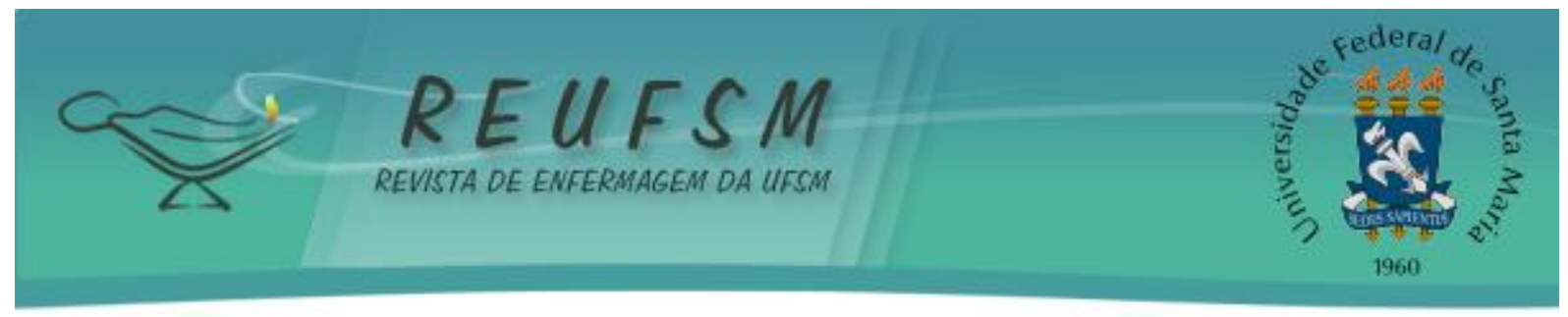

ARTIGO ORIGINAL

\title{
A CRISE HIPERTENSIVA E O CONHECIMENTO DA DOENÇA POR INDIVÍDUOS PORTADORES DE HIPERTENSÃO
}

\section{HYPERTENSIVE CRISIS AND KNOWLEDGE OF DISEASE PATIENTS WITH HYPERTENSION BY INDIVIDUALS}

\section{LA CRISIS HIPERTENSIVA Y EL CONOCIMIENTO DE LA ENFERMEDAD POR LOS INDIVIDUOS PORTADORES DE HIPERTENSIÓN}

Juliana Veiga Mottin da Silva ${ }^{1}$ Maria de Fátima Mantovani ${ }^{2}$

Elis Martins Ulbrich ${ }^{3}$

RESUMO: Objetivos: identificar os motivos para a busca do atendimento hospitalar na crise hipertensiva e analisar o conhecimento de indivíduos com hipertensão arterial sobre a doença. Metodologia: pesquisa descritiva, realizada em banco de dados do Grupo de Estudos Multiprofissional em Saúde do Adulto. Foi realizada a análise das entrevistas do banco de dados segundo análise temática de Bardin. Os participantes das entrevistas utilizadas foram doze que possuíam entre 18 a 65 anos, de ambos os sexos, com crise hipertensiva. Resultados: os dados propiciaram a construção de três categorias: 1- "Motivos apontados para a busca do atendimento hospitalar", 2-"Conhecimento da d'oença, que é caracterizada pelos fatores de risco e complicações", 3- "Formas de controle da doença". Considerações finais: os motivos para busca do serviço de saúde foram os sintomas e, o conhecimento da doença foi expresso através do reconhecimento da importância do controle dos hábitos de vida e das complicações.

Descritores: Hipertensão; Comportamento de busca de informação; Enfermagem.

ABSTRACT: Objectives: to identify why people look for hospital treatment during hypertension crisis and, to analyze the knowledge of people with hypertension about the disease. Methodology: descriptive research, held in the database of the Study Group on Multiprofessional Adult Health. The analysis of the interviews from the database was executed according to Bardin's subject analysis. The people interviewed were between 18 and 65 years-old, both male and female, with hypertensive crisis. Results: data propiciated the construction of three categories: 1- Reasons why patients search for hospital treatment, 2- Disease knowledge, characterized by risk factors and complications, 3- Ways to control the disease. Final Considerations: the reasons for seeking health care were the symptoms and the knowledge of the disease was expressed by recognizing the relevance to control life habbits and its complications.

Descriptors: Hypertension; Information seeking behavior; Nursing.

RESUMEN: Objetivos: identificar las razones para el atendimiento hospitalario en una crisis hipertensiva y analizar el conocimiento de individuos con hipertensión sobre la

\footnotetext{
${ }^{1}$ Enfermeira. Mestre em Enfermagem pelo Programa de Pós Graduação da Universidade Federal do Paraná (UFPR). Membro do Grupo de Estudos Multiprofissional em Saúde do Adulto (GEMSA). Curitiba, Paraná (PR), Brasil. Email: julianam.mottin6@gmail.com

2Enfermeira. Doutora em Enfermagem. Professora Associada da UFPR e do Departamento em Enfermagem da UFPR. Coordenadora da Iniciação Cientifica e Integração Acadêmica da UFPR. Bolsista Produtividade 2 CNPq, Membro do GEMSA. Curitiba, Paraná (PR), Brasil. Email: mfatimamantovani@ufpr.br

${ }^{3}$ Enfermeira. Mestre em Enfermagem. Doutoranda em Enfermagem da Universidade Federal do Paraná (UFPR). Bolsista REUNI. Membro do GEMSA. Curitiba, Paraná (PR), Brasil. Email: lilaulbrich@yahoo.com.br
} 


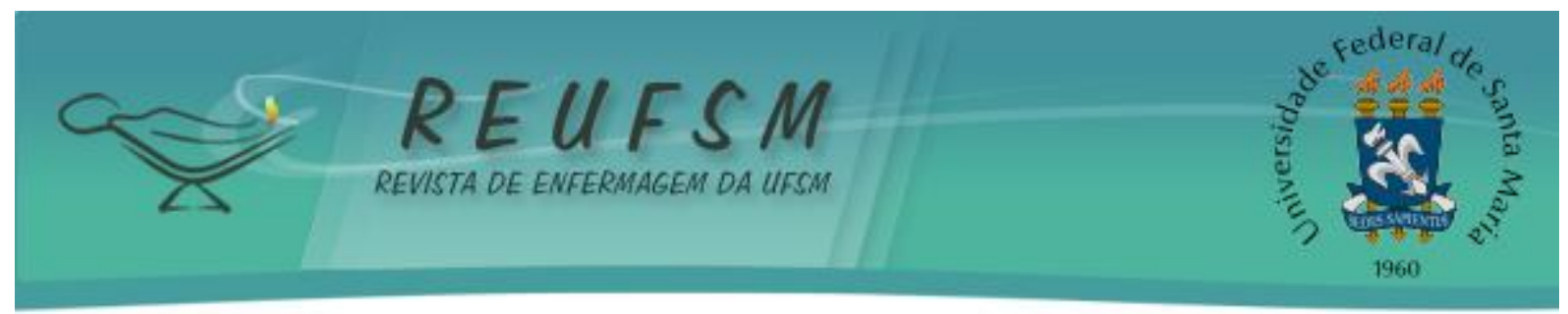

enfermedad. Metodología: investigación descriptiva, realizado en la base de datos del Grupo de Estudio Multiprofesional sobre la Salud del Adulto. Se realizó el examen de las entrevistas a partir de la base de datos de acuerdo con el análisis temático de Bardin. Fueron 12 participantes entre 18 y 65 años, de ambos sexos, con crisis hipertensiva. Resultados: los datos permitieron a la construcción de tres categorías: - "las razones para la búsqueda de la atención hospitalaria," - "El conocimiento de la enfermedad, que se caracteriza por factores de riesgo y complicaciones," - "Maneras de controlar la enfermedad". Conclusión: las razones para buscar atención médica fueron los síntomas y el conocimiento de la enfermedad fue expreso mediante el reconocimiento de la importancia de controlar los hábitos y las complicaciones.

Descriptores: Hipertensión; Conducta en la búsqueda de información; La Enfermería.

\section{INTRODUÇÃO}

As transformações ocorridas atualmente quanto à incidência e a prevalência das doenças, bem como quanto às principais causas de morte, demonstram que, no Brasil, os altos índices de óbitos causados por Doenças Crônicas Não Transmissíveis (DCNT) decorrem do estágio atual da transição demográfico/epidemiológica pela qual passa a população brasileira, resultando no envelhecimento populacional. ${ }^{1}$ Entre as DCNT estão as doenças cardiovasculares, que têm sido a principal causa de morte em nosso país, apresentando em 2010, 326.371 óbitos por doenças do aparelho circulatório. ${ }^{2}$

A hipertensão arterial (HA) é considerada um dos principais fatores de risco para - desenvolvimento das doenças cardiovasculares e representa a primeira causa de mortalidade no Brasil, sendo desde a década de 1960, mais comum que as doenças infecciosas e parasitárias. ${ }^{3-4}$ A característica multifatorial desta patologia associa-se frequentemente a alterações funcionais e/ou estruturais dos órgãos alvo (coração, encéfalo, rins e vasos sanguíneos) e a alterações metabólicas, com consequente aumento do risco de eventos cardiovasculares fatais e não fatais. ${ }^{5}$

Apesar de existirem programas específicos preconizados pelo Ministério da Saúde para o acompanhamento dos pacientes portadores de hipertensão e diabetes a elevada taxa de internamento por estas patologias ainda é preocupante. ${ }^{6}$

A crise hipertensiva é uma das formas de apresentação ou mesmo de complicação da hipertensão arterial, sendo didaticamente dividida em Urgência e Emergência, em estudo realizado anteriormente. ${ }^{7} \mathrm{Na}$ Urgência, o aumento da pressão arterial (PA) não representa risco imediato de vida e nem dano agudo a órgãos-alvo, portanto, o controle da PA pode ser feito, reduzindo-a gradualmente, em 24h. Em relação à Emergência, esta é a situação clínica caracterizada por PA marcadamente elevada e sinais de lesões de órgãosalvo requerendo internação hospitalar e imediato uso de drogas anti-hipertensivas parenterais, visando a redução da PA, em unidade de tratamento intensivo. ${ }^{7}$ No entanto, A Política Nacional de Atenção às Urgências adota somente o termo urgência, dividindo os atendimentos de urgências de maior ou menor gravidade ou complexidade. ${ }^{8}$

A dificuldade em encontrar dados epidemiológicos a respeito da crise hipertensiva é enfatizada em um estudo anterior, ${ }^{9}$ ao afirmar que a incidência/prevalência desta complicação é abordada muito precariamente na literatura e as referências são, geralmente, de estudos antigos ou realizados em populações pouco expressivas. Porém, a Urgência e a Emergência Hipertensiva são ocorrências clínicas, que podem representar mais de $25 \%$ dos atendimentos hospitalares de urgência. Estima-se que $3 \%$ de todas as visitas às salas de emergências são decorrentes de elevações significativas da pressão arterial. Usualmente, são situações clínicas que necessitam de redução mais rápida dos níveis pressóricos. ${ }^{9}$ 


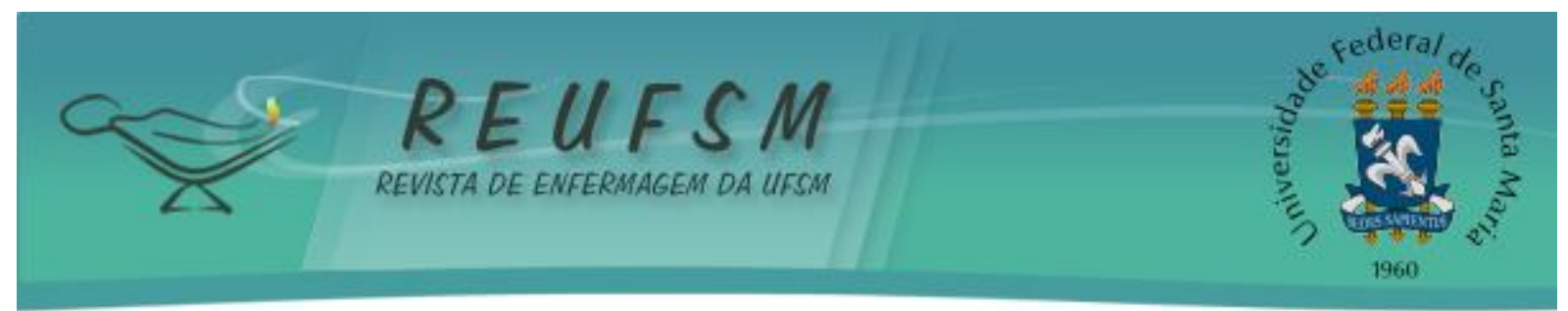

A internação por crise hipertensiva é, muitas vezes, o momento do reconhecimento do paciente quanto à sua condição de portador de uma enfermidade crônica e, o encontro do enfermeiro com as questões relativas ao adoecimento que este paciente apresenta.

Durante o desenvolvimento de pesquisa anterior a respeito do tema Hipertensão Arterial percebeu-se que muitos portadores desta patologia não entendem seu caráter crônico. Estes passam a aderir ao tratamento, a partir do momento que compreendem questões da doença e das complicações incapacitantes deste processo como o Acidente vascular cerebral (AVC) e a insuficiência renal, com consequente necessidade de realização de diálise/hemodiálise. ${ }^{10}$

Logo, justifica-se a importância deste estudo no ambiente hospitalar devido à crise hipertensiva ser uma das complicações da hipertensão arterial, visto que muitos pacientes passam a descobrir serem portadores desta patologia a partir do primeiro episódio da crise. Em estudo anterior ${ }^{11}$ comprovou-se que a hipertensão está entre as cinco principais causas de atendimento nos serviços de urgência e emergência.

Diante do exposto, os objetivos deste estudo foram: identificar os motivos para a busca do atendimento hospitalar na crise hipertensiva e, analisar o conhecimento de indivíduos com hipertensão arterial sobre a doença.

\section{METODOLOGIA}

A pesquisa é descritiva, realizada a partir de um banco de dados do Grupo de Estudos Multiprofissional em Saúde do Adulto (GEMSA). Os dados são secundários derivados de um macroprojeto desenvolvido em 2007. Foram realizadas entrevistas semiestruturadas de março a junho de 2007 para coleta de dados que ficaram sob a guarda dos pesquisadores. A Instituição selecionada como cenário para pesquisa foi um Hospital privado de Curitiba de pequeno porte, especializado no atendimento em Cardiologia de convênios e particulares, no setor de UTI Coronariana.

As entrevistas utilizadas para este artigo foram doze e os participantes da pesquisa eram adultos, que possuíam idade entre 18 e 65 anos de idade, de ambos os sexos e que procuraram o referido serviço com crise hipertensiva ou complicações associadas no período de realização do estudo. Foram abordadas questões referentes aos motivos para a busca do serviço de saúde, ao conhecimento da doença hipertensiva e aos mecanismos utilizados para o controle da patologia, além das questões referentes à caracterização dos portadores de hipertensão arterial.

Foram respeitadas as questões éticas e legais conforme a Resolução $n^{\circ}$ 196/96 do Conselho Nacional de Saúde, que regulamenta as pesquisas envolvendo seres humanos. ${ }^{12} 0$ projeto foi aprovado no Comitê de Ética e Pesquisa do Setor de Ciências da Saúde da UFPR, com CAEE: 0011.0.091.000-07, CEP/SD: 338.018.07.03, na data de 28 de março de 2007.

As entrevistas transcritas foram tratadas segundo a análise de conteúdo temático. ${ }^{13}$ Tema é a unidade de significação que se liberta naturalmente de um texto analisado segundo certos critérios relativos à teoria que serve de guia à leitura. É utilizado geralmente como unidade de registro para estudar motivações de opiniões, de atitudes, de valores, de crenças e tendências. ${ }^{13}$

A análise de conteúdo das entrevistas propiciou a construção de três categorias temáticas: 1- "Motivos apontados para a busca do atendimento hospitalar", 2"Conhecimento da doença: fatores de risco e complicações", 3-“Formas de controle da doença”. 


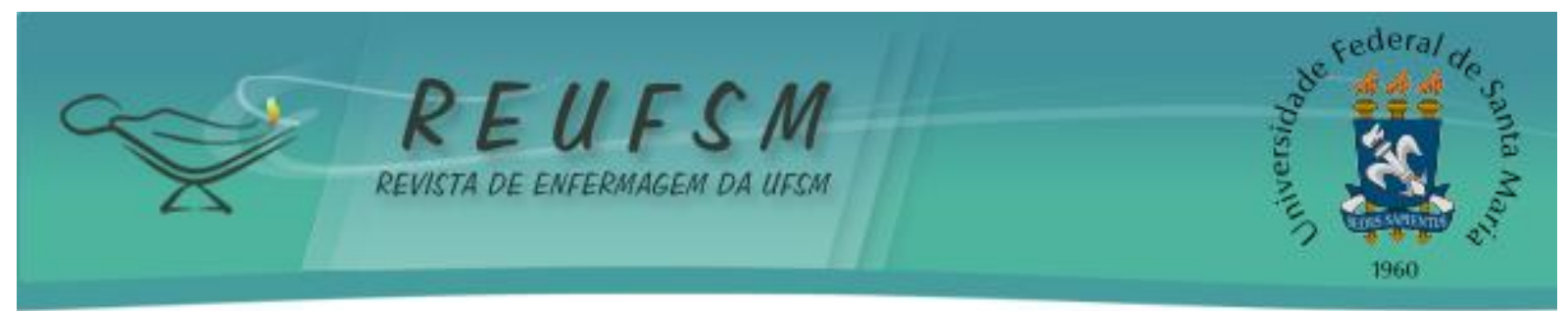

\section{RESULTADOS E DISCUSSÃO}

Dos 12 participantes, seis eram do sexo feminino e seis do sexo masculino, sendo que na amostra feminina as idades variaram de 33 a 60 anos e, na masculina, de 53 a 65 anos. Com relação ao estado civil, nove pacientes eram casados. 0 índice de escolaridade demonstrou que seis pacientes possuíam $3^{\circ}$ grau completo, três $2^{\circ}$ grau completo, um possuía $3^{\circ}$ grau incompleto e dois, $1^{\circ}$ grau completo.

$\mathrm{O}$ uso de medicamentos para controlar a HA foi citado por sete entrevistados, pois o restante da amostra não possuía diagnóstico de HA, sendo esta a primeira crise hipertensiva. Os dados demonstraram a relevância da observação de um estudo realizado que considerou ambos os casos da crise hipertensiva - emergência ou urgência, formas extremas de péssimo controle pressórico, que podem ser utilizadas como marcadores do cuidado inadequado com a doença hipertensiva. ${ }^{14}$

Cinco pacientes apresentaram pressão arterial diastólica (PAD) de $110 \mathrm{mmHg}$ ou superior, sendo considerada condição de crise hipertensiva e, estes mesmos pacientes desconheciam sua condição de portador de HA. Estes dados corroboram com estudo anterior $^{15}$ que, após realização de revisão de literatura, afirma que, grande parte dos portadores de hipertensão desconhece a patologia e mesmo diante do diagnóstico a maioria não realiza o tratamento de maneira adequada. ${ }^{15}$

Os cinco pacientes que desconheciam sua condição de portador de hipertensão contemplam a afirmação de que os eventos agudos das condições crônicas determinam uma busca por atenção, pois não é possível saber quando um episódio como este irá ocorrer, como uma emergência hipertensiva. ${ }^{16}$

Com relação à primeira categoria "Motivos apontados para a busca do atendimento hospitalar", dez entrevistados descreveram os seguintes sintomas: dor no peito, falta de ar, cefaleia, turvamento da visão, náusea, ansiedade e fraqueza; três referiram a dor no peito e outras não tão específicas como:

\section{[...] tive um chilique, agora faço exames preventivos [...]. (E1)}

[...] embaraçou um pouco a vista, senti um mal-estar com ânsia de vômito, dor atrás da cabeça [...]. (E7)

A sintomatologia referida da crise hipertensiva é de que esta inicia repentinamente e a pessoa pode apresentar: sensação de mal-estar, ansiedade, agitação, cefaleia sem causa definida, tontura, "borramento" da visão, dor no peito, tosse, apneia, náuseas, vômitos, palpitações, tontura, astenia, e outros. ${ }^{14}$

A procura pelo serviço de saúde acontece quando o paciente apresenta sintomas expressivos, que causam incômodos. A hipertensão é considerada uma "assassina silenciosa", pois muitos só apresentam sintomatologia quando o organismo alcançou seu grau máximo de estresse, estando na iminência de uma complicação. Quando esses sintomas aparecem, muitos sujeitos, como no caso descrito acima, consideram "chilique", não acreditando nos sinais que seu organismo exterioriza de situação alarmante interna.

No que se refere à segunda categoria "Conhecimento da doença: fatores de risco e complicações", as seguintes falas ilustram esta situação:

[...] sei que tem a ver com fumo, bebida, vida desregrada, alimentação, fazer esportes [...]. (E10) 


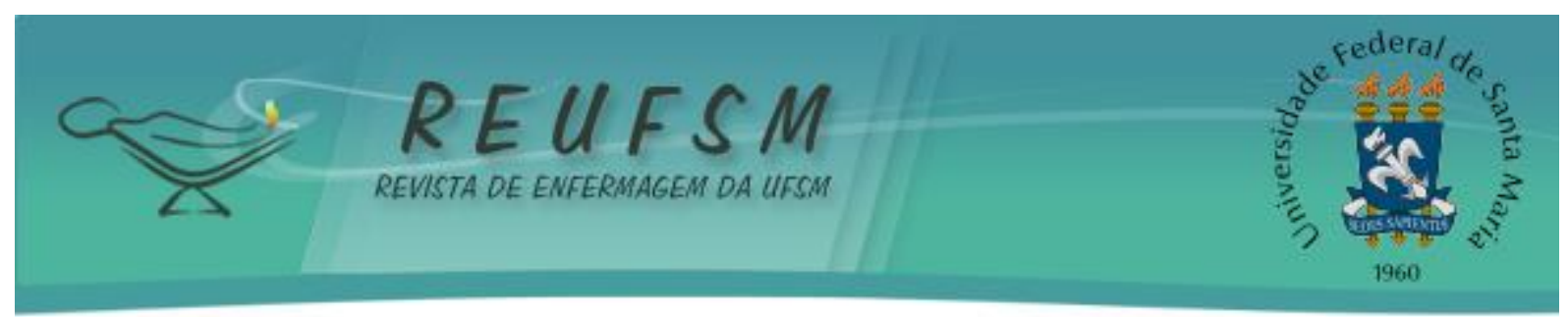

[...] é um dos caminhos para o infarto, para o derrame e outras complicações [...]. (E1)

Logo a doença não é reconhecida como tal, e sim por outras vertentes relacionadas às fontes de maior preocupação do portador de hipertensão, que são o controle dos hábitos de vida e as complicações que esta pode causar. Uma pesquisa anterior ${ }^{17}$ corrobora com estes dados, pois evidencia que os sujeitos tinham uma noção acerca dos fatores de risco da HA e de sua prevenção, oriundas do senso comum.

0 paciente, através da identificação das vertentes que representam uma ameaça à sua condição de vida, passa a entender que precisa se cuidar para que não ocorram consequências da doença que o impeçam de desenvolver suas atividades diárias.

Apesar da maioria dos pacientes ter referido já ter ouvido uma explicação sobre o que é a hipertensão arterial, estes passam a registrar na memória apenas o que precisam fazer: mudança de hábitos de vida e reconhecer indícios de complicações. Porém, um dos entrevistados reconheceu o caráter crônico da doença:

[...] é a pressão que aumenta, sei que é uma doença que se tratada pode ser controlada porque as pessoas não tratam, tenho até um aparelho em casa para medir, pelo que sei a gente pode controlar com exercício físico, alimentação $e$, estresse aumenta a pressão [...]. (E2)

Em estudo realizado anteriormente, os dados apontam que $78 \%$ da amostra selecionada não possuía conhecimento a respeito da hipertensão e os portadores que a descreveram o fizeram de forma incorreta. ${ }^{18}$ Logo se observa a compatibilidade com os dados da atual pesquisa, pois, dos 12 participantes, apenas um reconheceu que a hipertensão é uma doença crônica que necessita de controle constante. Este fato gera preocupação, pois, alguns pacientes passam a ter consciência da necessidade do controle dos níveis pressóricos, após vivenciar um episódio de crise hipertensiva, com sugestiva instalação de alguma complicação.

Em relação ainda às complicações, todos citaram o infarto e o acidente vascular cerebral, e apenas dois citaram a complicação renal.

[...] pode afetar o coração, rim, causar acidente vascular cerebral, essas coisas, muita pressão estoura tudo [...]. (E1)

Para qualquer doença crônica, o objetivo do tratamento é o seu controle, de modo a prevenir complicações, comorbidades e, sobretudo a mortalidade precoce. 0 alcance destes objetivos permite a melhoria da qualidade de vida com autonomia, se determinados tipos de complicações incapacitantes parcial ou totalmente forem evitados. ${ }^{19}$ Desta forma observa-se que há um conhecimento parcial ou total do risco da ocorrência de complicações da hipertensão ou da crise hipertensiva, porém, muitos pacientes entendem que este risco é iminente quando vivenciam um episódio de crise hipertensiva.

Um dos entrevistados citou a morte como uma complicação da hipertensão arterial. 0 pensamento da necessidade de uma vida regrada traz a preocupação antecipada da morte nos casos em que estes acabam saindo dessa rotina de controle.

[...] infarto na hora, aí vem a morte, que foi o que quase aconteceu comigo, sistema nervoso é o que atrapalha muito [...]. (E4) 


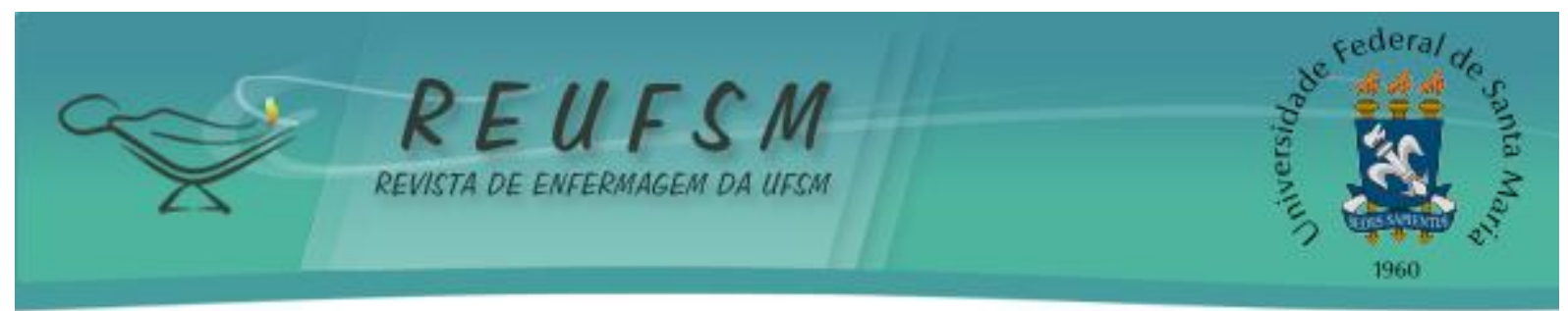

Esse pensamento sobre a iminência da morte pode gerar angústia e desconforto emocional para os pacientes, deixando-os muitas vezes, sem saber como agir diante do aparecimento de algum sintoma expressivo.

A terceira categoria "Formas de controle da doença", evidenciou que quando questionados a respeito do que fazer para evitar a crise hipertensiva, os pacientes consideravam não saber acerca da prevenção, não citando itens referentes à adesão ao tratamento. Porém, o controle dos fatores de risco prevaleceu novamente como resposta mais citada, apesar de não ser considerada como uma atitude de prevenção.

[...] não sei te dizer, sei que o sal aumenta, sei que o estado emocional aumenta, bebida alcoólica, essas coisas todas levam ao aumento da pressão [...]. (E1)

\section{[...] controlar a dieta, se estressar um pouco menos [...]. (E3)}

Alguns autores consideram que os sujeitos necessitam ampliar o conhecimento sobre os fatores de risco da HA, para preveni-la ou postergá-la. ${ }^{17}$ Porém, mesmo comprovados os benefícios do conhecimento da mudança sobre o estilo de vida, é mais fácil ao médico prescrever anti-hipertensivos do que orientar alterações de hábitos e de comportamentos, enquanto para os hipertensos é mais fácil tomar a medicação do que praticar exercícios físicos, fazer dietas, ou deixar de fumar. ${ }^{19}$ Isso significa que não só o hipertenso deve ser orientado com relação à sua doença, como também os profissionais de saúde em qualquer dos níveis de assistência, mas principalmente na atenção primária à saúde. Este fato pode ser observado na fala seguinte:

[...] tenho sido medicado normalmente, tenho feito exames, pelo tempo que vivo desse mal, já me acostumei, me convenci do que devo ou não devo fazer [...]. (E1)

Em algumas falas evidencia-se que, mesmo realizando tratamento para hipertensão, seus portadores continuam com dúvidas, o que favorece a ocorrência de crise hipertensiva como uma complicação da doença:

[...] uma dúvida que tenho é se ela pode ser controlada sem medicação, só com exercício e, se mesmo tratada essa hipertensão, ela pode dar complicação e, se com o tempo o funcionamento do coração fica prejudicado [...]. (E2)

[...] apesar de não consumir bebida alcoólica, reduzir sal, a pressão ainda tem picos de oscilação, será que se fizer mais exercício controla? (E6)

Essas dúvidas demonstram a lacuna de conhecimento que estes pacientes possuem, as quais são passíveis de orientação por parte da equipe de saúde. Assim, enfatiza-se a relevância do ato de orientar, que compete ao enfermeiro, na ocasião que precede a alta hospitalar e no acompanhamento pós-alta, em ambiente ambulatorial. ${ }^{20}$

Para que a orientação seja adequada à realidade da pessoa em seu meio social torna-se essencial repensar a formação acadêmica do enfermeiro. Esta deve valorizar a educação em saúde como dispositivo para a promoção da saúde e a prevenção de doenças. 


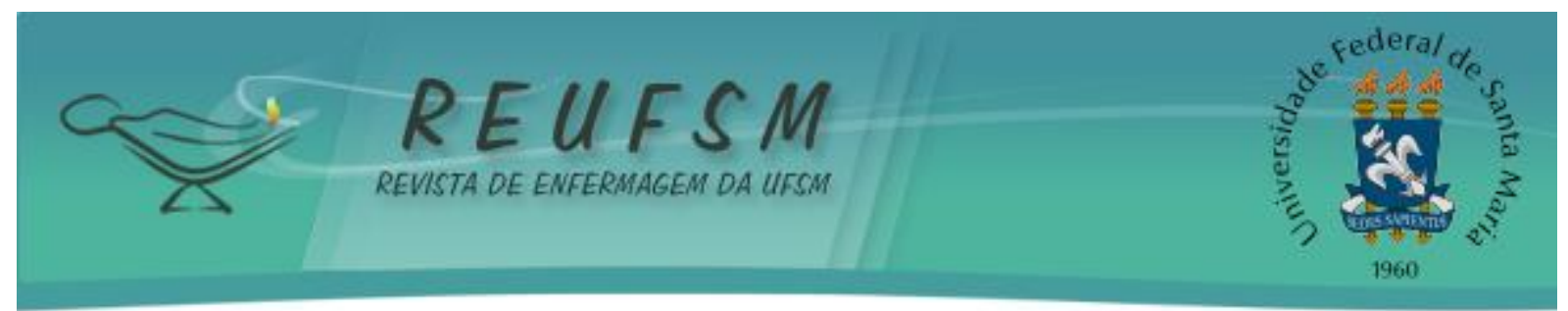

Assim, a formação de enfermeiros pode ser um fator contribuinte para que esta prática se reproduza junto aos usuários, suas famílias e a comunidade em que estão inseridos. ${ }^{21}$

\section{CONSIDERAÇÕES FINAIS}

A crise hipertensiva, além de ser uma consequência do descontrole da pressão arterial, muitas vezes, é o caminho para a descoberta da doença hipertensiva, conforme explicitado pelos dados analisados nesta pesquisa. Assim, os sintomas exteriorizados pelo organismo precisam ser valorizados, principalmente no momento da ocorrência da crise. Porém, muitos portadores de hipertensão desconsideram a importância deste fato, o que ocasiona maior probabilidade destes serem vítimas de uma complicação incapacitante da doença.

Os motivos para busca do serviço de saúde são os sintomas que, quando significativos, causam preocupação e receio aos portadores de HA. O conhecimento da doença foi expresso através do reconhecimento da importância do controle dos hábitos de vida e das complicações. 0 medo da morte surge como um risco iminente nos casos em que a doença é agudizada como na crise hipertensiva. Como formas de controle da doença emergiram o conhecimento e controle dos fatores de risco. Porém as outras formas de prevenção não foram citadas.

Os dados explicitam que, a maioria dos hipertensos conhece os fatores de risco da doença e suas complicações, porém, apesar desse conhecimento prévio, muitos não previnem as situações de emergência mediante a adesão ao tratamento. 0 enfermeiro, como cuidador e educador em saúde, deve orientar os pacientes que chegam ao serviço de emergência com crise hipertensiva a respeito da importância da investigação diagnóstica desta patologia, que é de fácil acessibilidade nas unidades básicas de saúde.

O conhecimento dos hipertensos acerca de sua patologia contribui para a compreensão da importância do tratamento. 0 fato de que, muitos pacientes desconhecem o caráter crônico da hipertensão é preocupante, sendo que muitos acreditam que esta doença pode ser curada, desconsiderando a importância do seu controle constante. A cura representa, para muitas pessoas, a concretização do sucesso no tratamento; no entanto, os portadores de hipertensão precisam estar cientes de que esta meta traduz-se na manutenção dos níveis pressóricos dentro dos padrões de normalidade.

Enfatiza-se que uma das limitações deste estudo foi a impossibilidade de analisar a adesão ao tratamento, devido a falta de informação fornecida por parte dos pacientes. Sugere-se a realização de outros estudos em instituições de saúde de emergência com características diferenciadas desta e que atenda a uma população que não apenas usuários de convênios e particulares. Com isto, almeja-se o encontro de maiores subsídios para realização de orientações e encaminhamentos adequados dos pacientes portadores de hipertensão arterial no sistema de saúde.

\section{REFERÊNCIAS}

1. Miranzi SSC, Ferreira FS, Iwamoto HH, Pereira G de A, Miranzi MAS. Qualidade de vida de indivíduos com diabetes mellitus e hipertensão acompanhados por uma equipe de saúde da família. Texto \& Contexto Enferm. Florianópolis. 2008 Out/Dez;17(4):672-9.

2. Brasil. Datasus. Ministério da Saúde [internet]. [acesso em 2012 Jun 23]. Disponível em: http://tabnet.datasus.gov.br/cgi/deftohtm.exe?hiperdia. 


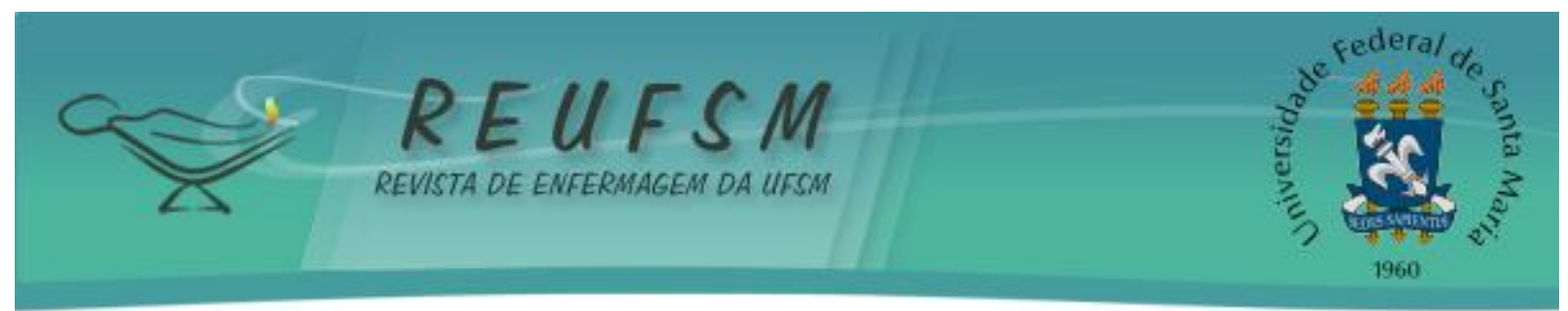

3. Brasil. Ministério da Saúde. Secretaria de Políticas de Saúde. Plano de Reorganização da Atenção à Hipertensão arterial e ao Diabetes mellitus. Manual de Hipertensão arterial e Diabetes mellitus. Brasília; 2002.

4. Pierin AMG. Hipertensão arterial: uma proposta para o cuidar. São Paulo: Manole; 2004.

5. Sociedade Brasileira de Cardilogia (SBC); Sociedade Brasileira de Hipertensão (SBH); Sociedade Brasileira de Nefrologia (SBN). VI Diretrizes Brasileiras de Hipertensão Arterial. São Paulo; 2010.

6. Brasil. Ministério da Saúde. Secretaria de Vigilância em Saúde. Departamento de Análise de Situação de Saúde. Plano de ações estratégicas para o enfrentamento das doenças crônicas não transmissíveis (DCNT) no Brasil 2011-2022. Brasília: Ministério da Saúde; 2011.

7. Furtado RG, Coelho EB, Nobre F. Urgências e emergências hipertensivas. Medicina, Ribeirão Preto. 2003 Abr/Dez [acesso em 2012 Jun 23];36:338-44. Disponível em: http://www.fmrp.usp.br/revista/2003/36n2e4/18urgencias_emergencias_hipertensivas.pdf.

8. Brasil. Ministério da Saúde. Portaria Conjunta N. ${ }^{\circ} 1.600$, de 7 de julho de 2011. Reformula a Política Nacional de Atenção às Urgências e institui a Rede de Atenção às Urgências no Sistema Único de Saúde (SUS). Legislações - GM

9. Martin JFV, Higashiama É, Garcia E, Luizon MR, Cipullo JP. Perfil de crise hipertensiva: Prevalência e apresentação clínica. Arq Bras Cardiol. 2004;83(2).

10. Mantovani MF, Mottin JV, Rodrigues J. Nurse Home Visity with Educational Activities in the arterial pressure treatment. Online Brazilian Journal of Nursing (OBJN- INSS 1676-4285) [internet]. $2007 \mathrm{Abr}$ [acesso em 2012 Maio 20];6(2). Disponível em: http: / / www.uff.br/objnursing/viewarticle.php?id=769

11. Jacobs CP; Matos EP. Estudo Exploratório dos Atendimentos em Unidade de Emergência em Salvador- Bahia. Rev Assoc Med Bras. 2005;51(6):348-53.

12. Conselho Nacional de Saúde. Resolução n. 196, de 10 de outubro de 1996. Dispõe sobre as diretrizes e normas regulamentadoras de pesquisa envolvendo humanos. Disponível em: www.saudeufpr.br.

13. Bardin L. Análise de conteúdo. Lisboa: Edições 70; 2010.

14. Franco RJS. Crise hipertensiva: definição, epidemiologia e abordagem diagnóstica. Rev Bras Hipertensão. 2002;9(4):340-5.

15. Souza FO. Hipertensão arterial sistêmica no município de Diogo de Vasconcelos - MG [monografia de especialização]. Minas Gerais: Universidade Federal de Minas Gerais; 2011. [acesso em 2012 Nov 23]. Disponível em: http://www.nescon.medicina.ufmg.br/biblioteca/imagem/2599.pdf

16. Mendes EV. O cuidado das condições crônicas na atenção primária à saúde: 0 imperativo da consolidação da estratégia da saúde da família. Brasília: Organização PanAmericana da Saúde; 2012.

17. Santos ZMSA, Lima HP. Tecnologia educativa em saúde na prevenção da hipertensão arterial em trabalhadores: análise das mudanças no estilo de vida. Texto \& Contexto Enferm (Florianópolis). 2008 Jan/Mar;17(1):90-7.

18. Mantovani MF, Mottin JV, Ulbrich EM, Pinotti S. Health clients profile and knowledge about arterial hypertension. Online Brazilian Journal of Nursing (OBJN- INSS 1676-4285) 


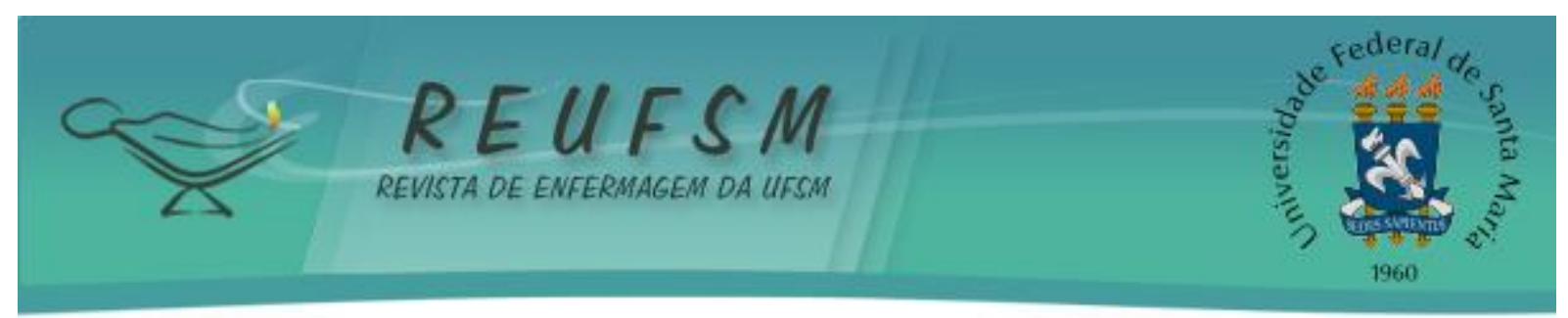

[internet]. 2008 [acesso em 2012 Abr 29];Abril 7(2). Disponível em: http: //www.uff.br/objnursing/viewarticle.php?id=769.

19. Lessa I. $O$ adulto brasileiro e as doenças da modernidade: epidemiologia das doenças crônicas não-transmissíveis. Editora Hucitec Abrasco: São Paulo/Rio de Janeiro; 1998.

20. Remonatto A, Coutinho AOR, Souza EM de. Dúvidas e expectativas de pacientes no pósoperatório de revascularização do miocárdio quanto à reabiltação pós-alta hospitalar: implicações para a enfermagem. Rev Enferm UFSM [internet]. 2012 [acesso em 2012 Out 10];Jan/Abr,2(1):39-48. Disponível em: http://cascavel.ufsm.br/revistas/ojs-

2.2.2/index.php/reufsm/article/view/3829

21 Silva LD da S, Beck CLC, Dissen CM, Tavares JP, Budó M de LD, Silva HS da. O enfermeiro e a educação em saúde: um estudo bibliográfico. Rev Enferm UFSM [internet]. 2012 [acesso em 2012 Nov 24]; Mai/Ago,2(2):412-9. Disponível em: http://cascavel.ufsm.br/revistas/ojs-

2.2.2/index.php/reufsm/article/view/2676/3769

Data de recebimento: $24 / 06 / 2012$

Data de aceite: 14/02/2013

Contato com autor responsável: Juliana Veiga Mottin da Silva

Endereço: Rua Professor Duílio Calderari, 2401, casa 02, Jardim Paulista - Campina Grande do Sul - PR - CEP: 83.430-000.

E-mail: julianam.mottin6@gmail.com 